\title{
OPTIMUM WATERMARK DESIGN BY VECTOR SPACE PROJECTIONS
}

\author{
Oktay Altun, Gaurav Sharma, Mark Bocko \\ ECE Dept., University of Rochester, Rochester, NY, USA
}

\begin{abstract}
We introduce an optimum watermark embedding technique that satisfies common watermarking requirements such as visual fidelity, sufficient embedding rate, robustness against noise and tolerance to benign signal processing, while optimizing one of these requirements. The algorithm distinguishes itself from other watermark optimization techniques in its flexibility for incorporating constraints and in assuring convergence to the globally optimum point when all constraints are convex. The proposed scheme is a natural extension of set-theoretic watermark design and inherits all the advantages of it. A watermarked image is first obtained by POCS, then optimum watermarked image is determined by an iterative search procedure based on a simple bi-section method. Experimental results are presented to illustrate the effectiveness of the method.
\end{abstract}

Index Terms - Optimum watermark embedding, set theoretic watermark, POCS, vector space projections, spreadspectrum watermark

\section{INTRODUCTION}

Digital watermarking is a special communication technique that utilizes digital multi-media files bearing perceptual information as the channel for the communication of watermark data. Watermarking is often the only solution available when secure out-band channels are not available for carrying the auxiliary information or when this information may be easily removed. Today watermarking is used in many areas including but not limited to copyright enforcement, tamper detection, broadcast monitoring and fingerprinting.

The exact requirements of watermark design depends on the specific needs of the application. However, we can talk about some common requirements for the design of watermarks in general. First of all, the watermark signals should be imperceptible, i.e., the watermarked media should look close to the original cover file. Secondly, the watermarking method should allow sufficient rate for the embedding. Depending on the application, the watermarks should also be able to withstand benign signal processing such as compression and/or malicious processing such as deliberate modifications.

This work was supported by the Air Force Research Laboratory/IFEC under grant number F30602-02-1-0129.
Several methods have been proposed falling into categories of suitably designed embedding $[1,2]$ that albeit requires ad hoc modifications for meeting application constraints, embedding with optimization of one criterion under other constraints $[3,4]$ and embedding by determining a feasible point meeting desired constraints. [5, 6] Among these methods, optimization based techniques are especially attractive because they allow minimization or maximization of a desired criterion subject to other constraints. Set theoretic methods on the other hand benefit form having a flexible and readily adaptable framework in which a wide variety of constraints can be incorporated. In addition, for convex constraints the methods assure global convergence.

In this paper we demonstrate how the set theoretic watermarking framework may be readily extended to an optimization based framework in which one of the constraint criteria may be selected for optimization. We do so using the method for quasi-convex optimization by convex feasibility problems. Although the optimization strategies look similar to [7], we employ more sophisticated visual models and robustness to compression in a simple framework.

\section{BACKGROUND}

\subsection{Common Constraints for watermarking}

The common requirements of watermarking such as imperceptibility, robustness to noise, robustness to compression and detectibility can be represented or approximated as convex sets $[5,6]$. These sets can be summarized as follows:

Detectibility: The watermarked image should give a positive response at the detector. Specifically for spread spectrum watermarks with the commonly used correlation detector, the correlation of the watermarked image with the key generated "watermark" pn-sequence should be greater than the threshold value. For $K$ different watermarks, we therefore have constraints:

$$
\mathbb{S}_{1}^{j} \equiv\left\{\mathbf{x}: \mathbf{w}_{j}^{T}(\mathbf{x}-\overline{\mathbf{x}}) \geq \tau_{j}\right\}, \quad j=1, \ldots, K .
$$

where $j$ denotes the watermark index, $\mathbf{w}_{j}$ denotes the watermark pn-sequence (assumed to be zero-mean), and ( $x$ denotes a candidate image; both being arranged as vectors in a chosen order. 
Visual Adaptation: The watermark should adapt the visual content of the file to maintain invisibility of the watermark noise. For this purpose, we employ constraints based on two models that capture different visual phenomena:

The first constraint is based on contrast sensitivity model. Human visual system is more sensitive to lower frequencies compared to higher frequencies. This fact has been explored and modelled by several researachers (e.g. Sakrison [8]) and results in the constraint set [6]:

$$
\mathbb{S}_{2} \equiv\left\{\mathbf{x}:\left\|\mathbf{H x}-\mathbf{H x}_{0}\right\| \leq \beta\right\}
$$

The second constraint exploits the fact that noise is less visible in textured regions compared to flat regions. A noise visibility function is modeled based on Pereira et al [3] and used to define a constraint set

$$
\mathbb{S}_{3} \equiv\{\mathbf{x}: \mathbf{l} \leq \mathbf{x} \leq \mathbf{u}\}
$$

where $l$ and $u$ are upper and lower bounds on pixel values that define the range of visually acceptable modification.

Robustness to compression: In a number of applications, the image is expected to be robust to benign signal processing operations. For spread spectrum watermarks and JPEG compression robustnes can be approximated as [6]:

$$
\begin{aligned}
\widehat{S}_{4} \equiv\left\{\mathbf{x}: w^{T}(I D C T\right. & \left(Q_{0}[D C T(\mathbf{x})]\right. \\
& \left.\left.\left.-\overline{I D C T\left(Q_{0}[D C T(\mathbf{x})]\right.}\right) \geq \gamma\right\}\right)
\end{aligned}
$$

where $Q_{0}[$ ] refers to the function that thresholds some (DCT) coefficients to zero and leaves others unchanged, the function being defined by coefficients quantized to 0 in JPEG compression of the image [5].

\subsection{Overview of Set Theoretic Watermarking and POCS}

Set theoretic watermarking [5,6] represents each property desired of the watermarked image as a constraint set. A watermarked image is then determined using an iterative algorithm that determines a point in the intersection of all the sets [9]. When the sets are convex the method of POCS provides a robust algorithm for this purpose. Given $n$ convex sets $\left\{S_{i}\right\}_{i=1}^{n}$ the POCS method determines a point in their intersection in the limit of projection sequence. If the intersection set is non-empty, the sequence $\left\{f_{k}\right\}_{k=0}^{\infty}$ generated by successive (relaxed) projections onto the sets converges to a point in the intersection, where

$$
f_{k+1}=\left(T_{S_{n}}\left(T_{S_{n-1}} \ldots T_{S_{1}}\left(f_{k}\right) \ldots\right)\right), k=0,1, . .
$$

$T_{S_{i}}=\left(1-\lambda_{S_{i}}\right) I+\lambda_{i} P_{S_{i}}, 0<\lambda_{i}<2$ is the relaxed projection operator onto set $S_{i}$. For unity relaxation $T_{S_{i}}\left(f_{k}\right)$ will be equal to $P_{S_{i}}\left(f_{k}\right)$, which is set to be in the rest of this paper [6]. Figure 1 schematically illustrates the POCS algorithm for two convex constraints.

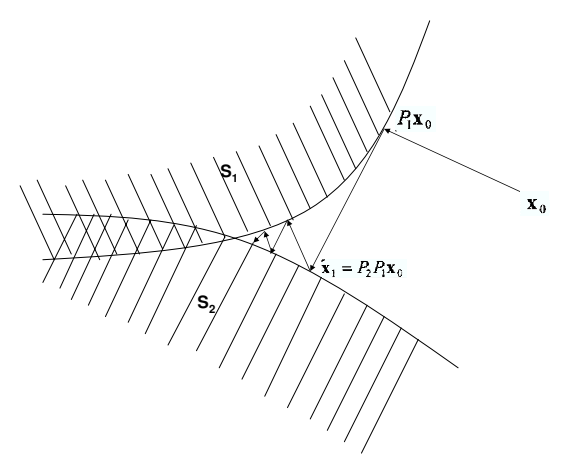

Fig. 1. A generic illustration of convergence of POCS algorithm as a solution of the feasibility problem for two sets.

\section{OPTIMUM WATERMARK EMBEDDING}

Common watermarking requirements can be formulated or approximated as convex constraints. If one of these requirements is desired to be optimized given the other requirements fixed, the objective function can be observed as a quasi-convex function where all of its sub-level sets are represented by convex inequalities. Hence the problem can be viewed as quasiconvex optimization problem [10, pp.159].

$$
\begin{array}{cl}
\min _{t} & \Phi_{t}(x) \\
\text { subject to } & \phi_{i}(x) \leq t, \quad i=1 . . m
\end{array}
$$

To be clear with the notation, let $\phi_{i}(x) \leq t$ represent the convex inequalities that represent the watermark requirements and $\Phi_{t}(x)$ be the family of one of these convex functions non-increasing in $t$. More precisely, $t$ represents embedding strength $\tau$ for $S_{1}, \beta$ for $S_{2}$, the control parameters $s_{0}$ and $s_{1}$ for $S_{3}$ and quality level $Q$ and $\gamma$ for $S_{4}$.

The set theoretic watermarking problem is a feasibility problem, quantitatively expressed as:

$$
\begin{aligned}
\text { find } & x \\
\text { subject to } & \phi_{i}(x) \leq t, \quad i=1 . . m \\
& \Phi_{t}(x) \leq 0
\end{aligned}
$$

Let $t^{*}$ denote the optimal value of quasi-convex optimization problem (7). On one hand, if there exist a solution for this problem, then we know $t^{*} \leq t$. On the other hand, if there is no solution for this feasibility problem then we know $t^{*} \geq t$. So the optimal value of this quasiconvex optimization function can be determined to be above or below the optimal point by solving the convex feasibility problem.

This observation is used as the basis of a simple algorithm for solving the optimization problem using bisection 
method, solving feasibility problem at each step. We assume two values of $t, u$ and $l$ are known, where $u$ parameter makes the solution of feasibility problem a null set and $l$ parameter makes the problem feasible. So we assume, $l<t^{*}<u$. We then solve the feasibility problem at $t=(l+u) / 2$, to determine whether the optimal value is in the upper or lower half of the interval. So this procedure generates an updated interval which has half the length of the previous length.

Bisection method for optimization of watermarks, given $l<t^{*}<u$, tolerance $\epsilon$

1. $t=(u+l) / 2$

2. solve feasibility problem (equation 7 ) by POCS

3. go step 1 until convergence criteria met, i.e. $u-l \leq \epsilon$

One practical solution of the feasibility problem in high dimensional signal spaces where one or some of constraints is formulated in transform domain is projection onto convex sets(POCS). The convergence of the algorithm to a feasible point on the boundary of a set is guaranteed when all constraints are convex. Also, it is possible to tell if the problem is feasible or not by examining the convergence of Lagrange multiplier calculated at each projection operation. In each iteration, the interval is bisected so that after exactly $\left\lceil\log _{2}(u-l) / \epsilon\right\rceil$ iterations, the algorithm will terminate.

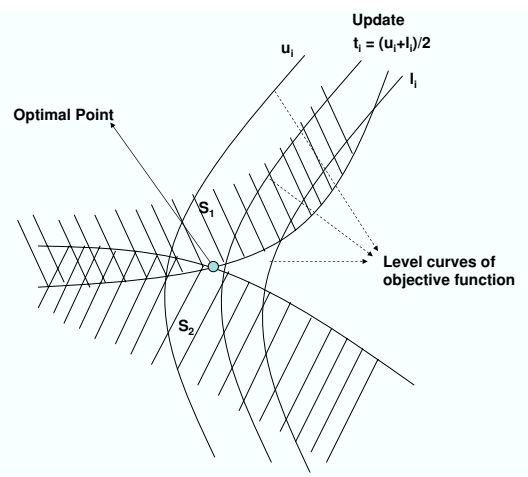

Fig. 2. A generic illustration of optimum watermarking by vector space projections.

\section{DIFFERENT OPTIMIZATION FORMULATIONS AND EXPERIMENTAL RESULTS}

Depending on the needs of the specific application, it is possible to formulate the optimization problem in different ways. In this section we will give various optimization scenarios and report the experimental results. We analyze some of the formulations and test our algorithm on various images from USC image database.
The convergence is checked by examining the convergence of Lagrange parameter at each projection. Convergence of Lagrange parameter below 0.1 is accepted as convergence criteria. In optimization of capacity values, some heuristic corrections are performed.

\subsection{Maximizing Embedding Strength}

The problem of watermark embedding can be formulated as maximizing the robustness/embedding stregth given a predetermined visual quality and robustness to compression. Table below summarizes the maximum embedding strength obtained by our algorithm.

The noise visibility function is controlled by two parameters $s_{0}$ and $s_{1}$ [3] , and we assume $s_{1}=10 \cdot s_{0}$ all the times. The pn-sequence is image size and mean corrected correlation detector described in equation (1) is employed.

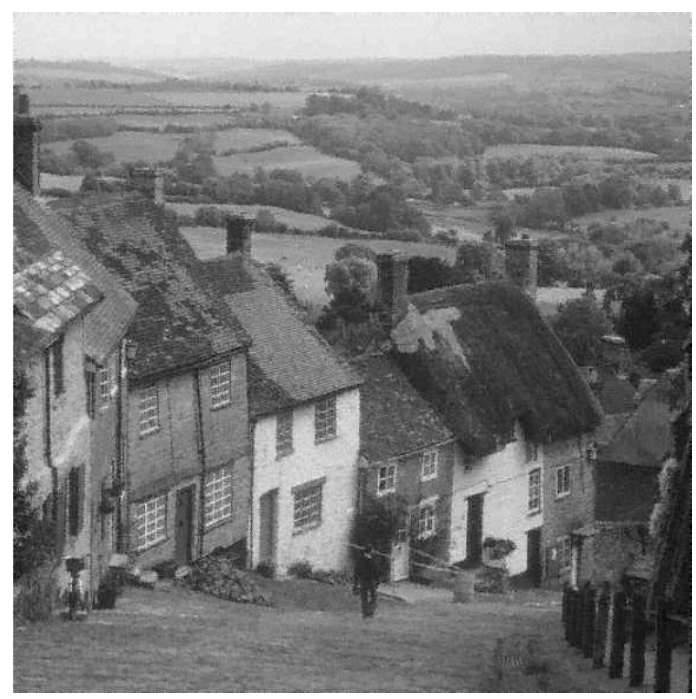

Fig. 3. Goldhill image watermarked by maximization of watermark strength. $\left(s_{0}=2\right)$

Table 1. Summary of watermark optimization results for Goldhill image in the USC Image database. The maximum watermark strength, at various visual quality levels are reported.

\begin{tabular}{|c|c|c|c|}
\hline & $s_{0}=3$ & $s_{0}=2$ & $s_{0}=1$ \\
\hline 1 bit & 12.46 & 8.32 & 4.7 \\
\hline
\end{tabular}

\subsection{Maximizing Embedding Rate}

In this strategy, watermark designer aims to embed maximum data given a fixed embedding strength, fidelity and robustness 
to compression. We report optimization results for those cases and report the results for Goldhill image.

Table 2 gives the maximum amount of information embedded into the Goldhill image at various embedding strengths and fidelity.

Table 2. Summary of capacity optimization results for Goldhill image in the USC Image database. The maximum capacity at various embedding strengths and visual quality levels are reported.

\begin{tabular}{|c|c|c|c|}
\hline & $s_{0}=3$ & $s_{0}=2$ & $s_{0}=1$ \\
\hline$\tau=0.5$ & 291 & 183 & 37 \\
$\tau=1$ & 118 & 42 & 12 \\
$\tau=2$ & 23 & 12 & 3 \\
\hline
\end{tabular}

\subsection{Minimizing distortion}

In some other applications, it may be desirable to minimize distortion given certain fidelity and robustness. We report optimization results for those cases and tabulate the results for various images. The images show different optimum capacity values depending on their visual complexity.

Table 3. Summary of visual fidelity minimization based on (noise visibility function) results for Goldhill image in the USC Image database. The minimum visual distortion (summarized by $s_{0}$ parameter), at various bit and embedding strength levels are reported.

\begin{tabular}{|c|c|c|c|}
\hline & $\tau=0.5$ & $\tau=1$ & $\tau=2$ \\
\hline 1 bit & 0.109 & 0.232 & 0.492 \\
10 bits & 0.462 & 0.876 & 2.458 \\
100 bits & 1.864 & 3.945 & 13.443 \\
\hline
\end{tabular}

\section{CONCLUSION}

We demonstrate an optimum watermark insertion technique based on vector space projections. The algorithm requires convex set formulation of common watermarking requirements. A bisection search algorithm along with the vector space projections, both assuring convergence, provides a globally convergent optimum watermark solution. The algorithm inherits all advantages of set theoretic formulation, main advantage being the flexible formulation of the problem. Possible variations of optimization algorithms are tested, such as minimization of visual distortion, maximization of robustness, maximization of capacity etc. The algorithm is relatively slow in nature due to several set theoretic steps. In future work, we plan to employ more sophisticated methods to predict the convergence of each feasibility problem by fitting an exponent to the convergence of Lagrange parameter at each projection.

\section{REFERENCES}

[1] B.Chen and G.W.Wornell, "Quantization index modulation: a class of provably good methods for digital watermarking and information embedding," IEEE Trans. Inform. Theory, vol. 47, pp. 1423-1443, 2001.

[2] I. Cox, J.Kilian, F.T.Leighton, and T.Shamoon, "Secure spread spectrum watermarking for multimedia," IEEE Trans. Image Proc., vol. 6, no. 12, pp. 1673-1687, Dec. 1997.

[3] S. Pereira, S. Voloshynoskiy, and T. Pun, "Optimal transform domain watermark embedding via linear programming," Signal Processing, vol. 81, no. 6, pp. 12511260, Jun. 2001.

[4] M. K. Mihcak, R. Venkatesan, and M. Kesal, “Optimization algorithms for quantizing randomized statistics of image regions," in Proceedings of the Fortieth Annual Allerton Conference on Communication, Control and Computing, Oct. 2002.

[5] O. Altun, G. Sharma, M. Celik, and M. Bocko, "Set theoretic watermarking and its application to semifragile tamper detection," IEEE Trans. Information Forensics And Security, August 2005, submitted for review.

[6] O. Altun, G. Sharma, M. Celik, and M. Bocko, "Semifragile hierarchical watermarking in a set theoretic framework," in Proc. of ICIP, 2005, vol. 1, pp. 10011004.

[7] M. L. Miller, I. J. Cox, and J. A. Bloom, "Informed embedding: Exploiting image and detector information during watermark insertion," Proc. of ICIP, 2000.

[8] J. L. Mannos and D. L. Sakrison, "The effects of a visual fidelity criterion on the encoding of images," IEEE Transactions on Information Theory, vol. 20, no. 4, pp. 525-536, Jul. 1974.

[9] P. L. Combettes, "The foundations of set theoretic estimation," Proceedings of the IEEE, vol. 81, no. 2, pp. 182-208, Feb. 1993.

[10] S. Boyd, "Convex optimization," Cambridge University Press, http://www.stanford.edu/ boyd/cvxbook/. 